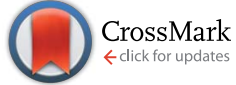

Cite this: RSC Adv., 2015, 5, 57328

Received 9th May 2015

Accepted 23rd June 2015

DOI: $10.1039 / \mathrm{c} 5 \mathrm{ra0} 8643 \mathrm{~d}$

www.rsc.org/advances

\title{
Delamination of graphite in a high pressure homogenizer $\dagger$
}

\author{
T. J. Nacken, C. Damm, J. Walter, A. Rüger and W. Peukert*
}

A scalable industrial method for graphene and Few-Layer-Graphene (FLG) production by graphite delamination in $\mathrm{N}$-methylpyrrolidone and water-surfactant mixtures using a high pressure homogenizer is presented. This paper is focused on processing conditions and extensive subsequent analysis of the delaminated products by a combination of analytical ultracentrifugation, UV/Vis and statistical Raman spectroscopies including co-localization with atomic force microscopy. In this way quantitative processing-structure-property correlations showing how suspension properties and processing parameters governing yield, quality and lateral dimension of the produced graphene are obtained. It is found that a high pressure homogenizer can be used to obtain sufficiently high concentrated FLG suspensions with low defect concentration.

\section{Introduction}

Graphene is a 2D-sheet material consisting of a one atomic layer of $\mathrm{sp}^{2}$-hybridized carbon. Since its first discovery graphene has become one of the most investigated materials in the new millennium, because of its outstanding physical properties, e.g. ballistic electron transport and high charge carrier density. ${ }^{\mathbf{1 - 8}}$ Within the last decade different graphene production methods have been established. These methods can be divided into bottom-up (e.g. chemical vapor deposition) and top-down approaches, namely ultrasonication, ${ }^{9-11}$ dry or wet planetary ball milling, ${ }^{\mathbf{1 2 - 1 5}}$ stirred media delamination ${ }^{\mathbf{1 6 - 1 8}}$ and high shearmixing in rotor-stator devices ${ }^{\mathbf{1 9 - 2 1}}$ in either organic solvents or water-surfactant mixtures. Today, even combination of techniques is known, e.g. planetary ball milling or microwave treatment and subsequent ultrasonication. ${ }^{\mathbf{1 4 2 2}}$ Furthermore large-scale production of graphene oxide like compounds in aqueous media is possible by electrochemical graphite exfoliation. ${ }^{23}$ Drawbacks of graphene production by ultrasonication and planetary ball milling techniques are limited scalability, long processing times and therefore low production rates.

In this paper an industrial high pressure homogenizer (HPH) is used as delamination device for delamination of graphite suspensions. This method is simple, scalable and does not require toxic chemicals, graphite oxidation or ultrasound postprocessing to achieve exfoliation. Moreover, due to residence times in the nozzle $<1 \mathrm{~s}$ HPH provides mild exfoliation conditions which helps to avoid defect formation. HPH devices are

Institute of Particle Technology (LFG), Friedrich-Alexander University Erlangen-Nürnberg (FAU), Cauerstrasse 4, 91058 Erlangen, Germany. E-mail: wolfgang.peukert@fau.de; Tel: +49-9131-85-29401

$\dagger$ Electronic supplementary information (ESI) available. See DOI: 10.1039/c5ra08643d commonly used in food industry, cell disintegration, ${ }^{24}$ emulsification $^{25,26}$ or dispersion of active pharmaceutical ingredients. ${ }^{27,28}$ Recently, HPH devices were employed for graphene production by exfoliation of thermally reduced graphite oxide. The produced graphene dispersions were used for manufacturing graphene films by printing techniques or for preparation of graphene nanocomposites with superior mechanical and electrical properties. ${ }^{29-31}$ Processing of unmodified graphite by HPH, however, did not yield stable graphene suspensions. ${ }^{31}$ Yi et al. showed the feasibility of delamination by HPH for unmodified graphite and other layered materials and investigated the influence of feed concentration and processing time on yield and morphology of the product. ${ }^{32-35}$ Moreover, Yi et al. demonstrated $10 \mathrm{~L}$ batch graphene production by a jet cavitation method and obtained few layer graphene with low defect concentration. ${ }^{36}$ The processing time, however, was quite long $(8 \mathrm{~h}) \cdot{ }^{36}$ In this paper we report an environmental friendly method for graphene production in large quantities ( $5 \mathrm{~L}$ batches) in processing times $<3 \mathrm{~h}$ by top-down processing of isostatic and unmodified graphite in an industrial high pressure homogenizer. In contrast to the device used by Yi et al. our device allows a simple control of the nozzle pressure by adjusting the flow rate and a controlled suppression of cavitation by applying a counter pressure. The graphite delamination process is performed in aqueous dispersions of the non-ionic surfactant TWEEN@80 (TW80) as an inexpensive, non-toxic and therefore environmental friendly dispersing medium. We selected TW80 as surfactant because according to preliminary studies for the used graphite it gave the best exfoliation result amongst the investigated commercial surfactants. We further focused on advanced analytical methods to study the influence of processing conditions on yield and quality of the product in detail. Usually exfoliated graphene sheets are analyzed by Transmission Electron Microscopy (TEM) and/or Atomic Force 
Microscopy (AFM). In TEM, however, only a few $\mu \mathrm{m}^{2}$ of the sample area are investigated and flakes with very small lateral dimensions can get lost during sample preparation because of using holey grids as sample supports. AFM gives a more representative image of the flake size distribution than TEM because larger sample areas can be scanned and there is no loss of any particle size fraction during sample preparation. The measured flake height, however, is influenced by adsorbed surfactant and the sample preparation/washing procedure. Thus, we focus on statistical Raman spectroscopy which is less influenced by adsorbed surfactants and allows investigation of a large sample area $\left(\geq 3600 \mu \mathrm{m}^{2}\right)$. Further, statistical Raman spectroscopy is combined with AFM and also with analytical ultracentrifugation (AUC) to get a highly detailed picture of the product quality regarding delamination degree, particle size distribution and defects.

Statistical analysis of more than 1300 individual Raman spectra per sample provides representative insight into the product quality distribution with respect to in-plane defects, degree of delamination (flake thickness) and lateral flake dimension..$^{17,18,37,38}$ In addition, AUC provides detailed insights into the sedimentation behaviour of the obtained platelet suspensions and allows the determination of lateral dimensions directly in solution, if the flake height is known. ${ }^{39}$

\section{Experimental}

\section{Materials}

The unmodified isostatic graphite GSI70 (RMC Remacon $\mathrm{GmbH}$, Germany) was used as feed material for the delamination experiments. It has a purity $>99.5 \%$ and a median particle size $x_{50,3}$ of $15-20 \mu \mathrm{m}$ (measured by the supplier using a Cilas particle sizer 920). In addition the unmodified natural graphite "GNP6" (RMC Remacon GmbH, Germany) was used to demonstrate transferability to a different feed material. It has a purity $>99.5 \%$ and a median particle size $x_{50,3}$ of $3.5 \mu \mathrm{m}$ (measured by the supplier using a Cilas particle sizer 920). In standard suspensions the non-ionic surfactant TWEEN®80 (TW80) (Sigma-Aldrich GmbH, Germany) was used and for transferability the anionic surfactant sodium dodecyl sulfate (SDS) (Sigma-Aldrich GmbH, Germany) was used to prevent agglomeration of the graphene particles obtained by graphite delamination in water. $N$-Methylpyrrolidone (NMP) was purchased from Merck with a purity $>99.5 \%$. All materials were used as supplied without further purification. Deionized water was used for preparation of all graphite suspensions.

\section{Delamination experiments}

For delamination experiments graphite suspensions in aqueous solutions of the surfactant TW80 or SDS were prepared. All delamination experiments were carried out in a high pressure homogenizer "Economic Dispersionizer Omega®60" (Netzsch Feinmahltechnik GmbH, Germany). In this device the graphite suspension is pumped through a nozzle with a defined flow rate to adjust the system pressure. The principle of high pressure homogenization is shown schematically in Fig. 1.

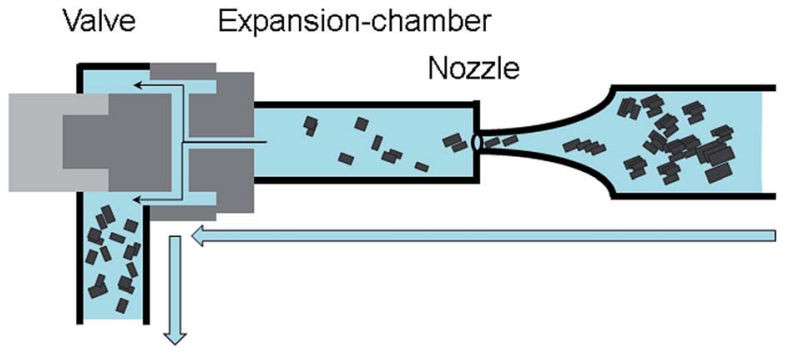

Fig. 1 Principle of graphite delamination by HPH. The suspension is pumped through a nozzle and released into an expansion-chamber. A counter pressure can be applied to the suspension by closing the valve after the expansion chamber.

The Omega ${ }^{\circledR 60}$ is equipped with a $15 \mathrm{~L}$ double walled stirred tank connected with a thermostat for feed suspension holdup and cooling. Inside the tank the suspension was stirred with $1000 \mathrm{rpm}$ using a blade stirrer for pre-homogenization. Processing can be performed in continuous mode or batch mode. For the presented study only the batch mode was chosen. The pressure was varied in the range from 10 to $70 \mathrm{MPa}$ by adapting the flow rate through the nozzle with a diameter of around $240 \mu \mathrm{m}$.

The batch mass was kept constant at $5 \mathrm{~kg}$ for each experiment. Graphite feed suspensions were prepared in two steps. First the desired amount of surfactant was dissolved in water to adjust the targeted surfactant concentration. $4.95 \mathrm{~kg}$ of the water surfactant mixture were transferred to the feed tank of Omega ${ }^{\circledR 60}$ and stirred for cooling until $18{ }^{\circ} \mathrm{C}$ was reached. In the second step the desired amount of graphite powder $(50 \mathrm{~g})$ was added into this tank under stirring for pre-dispersing. For each pressure the graphene production was followed over six batch runs.

Samples for subsequent analyses were taken after each batch run and centrifuged using a "Centrifuge Sigma 3-30KS" (Sigma $\mathrm{GmbH}$, Germany) to remove not yet delaminated feed particles from the suspensions prior to further characterization. The used centrifugation conditions correspond to a "cut size" for sedimentation of a sphere-equivalent diameter of $400 \mathrm{~nm}$ (see $\mathrm{ESI} \dagger)$. The cut size is calculated using the Stokes equation (ESI $\dagger)$. It is used instead of the commonly used time and rotational speed for centrifugation to show clearly which particle sizes are separated from the product. Post-processing is a crucial step that deserves more attention, also compare Damm et $a{ }^{1{ }^{17}}$ and Nacken et al. ${ }^{18}$

\section{Product characterization}

Thermo-gravimetric analysis (TGA). TGA was performed in synthetic air using a "TGA Q50" (TA Instruments) to determine the graphene content of a dried mass from processed suspensions. The heating rate was $10 \mathrm{~K} \mathrm{~min}^{-1}$ and the temperature was hold at 150, 450 and $1000{ }^{\circ} \mathrm{C}$, for 30,180 and 30 minutes, respectively. The results of TGA allowed us to distinguish between mass loss due to evaporation of residual water, surfactant decomposition (up to $400{ }^{\circ} \mathrm{C}$ ) and decomposition of produced graphene $\left(520-1000{ }^{\circ} \mathrm{C}\right.$ ) (for details see ESI $\dagger$ ). 
UV/Vis-spectroscopy. UV/Vis-spectra of the suspensions were recorded using a "Cary 100 Scan" spectrophotometer (Varian) in the wavelength range from $350 \mathrm{~nm}$ to $800 \mathrm{~nm}$ using quartz cuvettes with an optical path length of $1 \mathrm{~cm}$.

Wafer coating. For Raman spectroscopy and Atomic Force Microscopy (AFM) $15 \mu \mathrm{L}$ of the supernatant of centrifuged delaminated samples were deposited on a silicon wafer with a $300 \mathrm{~nm}$ thick $\mathrm{SiO}_{2}$ layer on top by drop-coating. Prior to coating all samples were diluted with the corresponding liquid to yield an absorbance of 0.1 for $660 \mathrm{~nm}$. The wafers were heated while coating, dried at ambient temperature for $24 \mathrm{~h}$ and then washed with water and ethanol to remove excess surfactant, which disturbs subsequent analysis particularly for AFM measurements.

Statistical Raman spectroscopy. Raman spectra were recorded in the wave number range from $800 \mathrm{~cm}^{-1}$ to $3300 \mathrm{~cm}^{-1}$ using a confocal Raman spectrometer "LabRAM HR Evolution" (Horiba). Spectra were recorded using a $100 \times$ objective (laser spot size $\sim 0.72 \mathrm{~nm}$ ) and $532 \mathrm{~nm}$ Nd-YAG laser (second harmonic). The laser beam was attenuated to $50 \%$ of its original intensity using a filter and the integration time was set to $5 \mathrm{~s}$ per recorded data point to prevent extended heating of the sample. For scanning probe microscopy a automated movable table (Märzhäuser) for two-dimensional maps was used (minimal step size $0.1 \mu \mathrm{m})$.

Statistical Raman spectroscopy was performed by creating a square shaped map with spacing of $1.4 \mu \mathrm{m}$ between two adjacent data points in each $x$ - and $y$-direction. In this way overlap of data points is prevented. At each point a Raman spectrum was taken. In this work Raman maps spanning a minimum square of $60 \times 60 \mu \mathrm{m}^{2}$ equal to 1369 points or $70 \times 70 \mu \mathrm{m}^{2}$ equal to 2601 individual points were recorded. For evaluation the D-, Gand 2D-Raman peaks were fitted by single Lorentz-functions using the software LabSpec 6.3.39.2 to determine height and full width at half maximum (FWHM) of the peaks. Statistical evaluation was performed by analysis of all obtained Raman spectra with G-peak intensities $>500$ counts. Note: only statistics containing a minimum of 200 evaluated spectra are presented in this paper.

Atomic force microscopy (AFM). AFM images were recorded on a NanoWizard ${ }^{\circledR} 3$ (JPK Instruments, Berlin, Germany). If not stated otherwise for each image a surface of $5 \times 5 \mu \mathrm{m}^{2}$ with a resolution of $1024 \times 1024$ pixels $^{2}(\sim 4.9 \mathrm{~nm}$ per pixel) was recorded. Imaging was performed in intermittent contact mode with a NSC15/AlBS probe (frequency $325 \mathrm{kHz}$, spring constant $46 \mathrm{~N} \mathrm{~m}^{-1}$ and nominal tip radius $<10 \mathrm{~nm}$ ). All obtained AFMimages were analyzed by contrast imaging in Gwyddion 2.36. To identify the graphene flakes a threshold value for the height was set corresponding to $5-15 \%$ contrast. Flakes or flake-like structures with lateral dimensions of less than $10 \mathrm{~nm}$ disc equivalent radius were neglected for evaluation as the error caused by the used cantilever (radius) would be significant. Also flakes with mean height of $>50 \mathrm{~nm}$ were neglected as these flakes are agglomerates formed by solvent evaporation during sample preparation process.

Co-localized statistical Raman spectroscopy and AFM imaging. To ensure that precisely the same area on the wafer was measured by Raman spectroscopy and AFM, both instruments were synchronized using a transportable sample-stage. The stage can be moved mechanically in $x$ - and $y$-direction. Firstly, an AFM map over a reference glass slide was created to align the cantilever position of the AFM probe with the crosshair and thus the stage. Then the stage was carefully demounted and put onto the automated movable table (Märzhäuser) of the Raman spectrometer. Without moving the stage itself the laser was positioned onto the crosshair using the automated table. Finally the reference glass slide was exchanged by the real sample. The map size was set to $20 \times 20 \mu \mathrm{m}^{2}$. The spacing for statistical Raman spectroscopy between two data points in each $x$ - and $y$-direction was set to $0.25 \mu \mathrm{m}$ to guarantee a complete surface scan without vacancies.

Analytical ultracentrifugation (AUC). A preparative centrifuge, type Optima L-90K from Beckman Coulter, U.S.A., has been modified with a multiwavelength (MWL) absorbance detector to be used for the sedimentation velocity (SV) experiments. Detailed information regarding the MWL optics as well as the custom data acquisition software are available in ref. 40 and 41. For all experiments we used titanium centerpieces manufactured by Nanolytics, Germany, with path lengths of 12 $\mathrm{mm}$ in combination with sapphire windows.

Sedimentation velocity data was acquired at $4000 \mathrm{rpm}$ and $20{ }^{\circ} \mathrm{C}$ in water. The solvent density was $998.3 \mathrm{~kg} \mathrm{~m}^{-3}$ and the viscosity was $1.003 \mathrm{mPa}$ s. Intensity data was recorded for all chambers individually and was converted after the experiment to absorbance data using the region above the sample's meniscus (airregion) in the measurement cell. The absorbance data at $400 \mathrm{~nm}$ was fitted to the least-square based ls- $g *(s)$ direct boundary distribution model using Sedfit 14.1. The apparent and not diffusion corrected sedimentation coefficient distribution $g^{*}(s)$ is obtained by fitting the data to the direct solution of the radial and temporal concentration profile in the measurement cell. ${ }^{42}$ The data were fitted with a second derivative regularization, a resolution of 100 grid points and fitting ranges of 10 to 120000 sved ( 1 sved equals $10^{-13}$ seconds) depending on the sample. Time-independent, radial invariant noise, as well as meniscus positions were fitted.

Lateral size distributions were obtained from the sedimentation coefficients applying the methodology presented previously. ${ }^{39}$ Since no exact material parameters exist or could be measured for TW80 stabilized few-layer-graphene, we estimated these values allowing for a rough approximation of the lateral sizes. A density of $2000 \mathrm{~kg} \mathrm{~m}^{-3}$ was used for the graphene layers and the density of the shell was estimated to be equal to the solvent density. The graphene layer thickness used for the calculations was $1 \mathrm{~nm}$ for 1-layer graphene, $2.4 \mathrm{~nm}$ for 3-layer graphene and $3.8 \mathrm{~nm}$ for 5-layer graphene. An enlarged shell thickness of $1.8 \mathrm{~nm}$, taking into account the stabilizer bound to the graphene surface, was used for all calculations.

\section{Results and discussion}

\section{Production yield $v s$. processing parameters}

The first important information for any technically relevant production method is the obtained mass of product and the related production rate. To calculate the concentration from the 
UV/Vis-absorbance using the Lambert-Beer law the extinction coefficient for the material must be known. In literature (for $\lambda=$ $660 \mathrm{~nm}$ ) different values for the extinction coefficient are found, which range from $1390-6600 \mathrm{~L} \mathrm{~g}^{-1} \mathrm{~m}^{-1}$ dependent especially on the product post-processing by centrifugation and production method., ${ }^{\mathbf{9} 3 \mathbf{4 3 4}}$ Thus, we determined the extinction coefficient for our material as described in the ESI $\dagger$ and obtained a value of $1028 \mathrm{~L} \mathrm{~g}^{-1} \mathrm{~m}^{-1}\left( \pm 83 \mathrm{~L} \mathrm{~g}^{-1} \mathrm{~m}^{-1}\right)$. Applying the found extinction coefficient, the dispersed carbon concentration was determined for each processing parameter by measuring the absorbance at $660 \mathrm{~nm}$. Evaluation of preliminary experiments via UV/Visspectroscopy showed that the non-ionic surfactant TW80 is a better stabilizer for carbon than the anionic surfactant SDS (see ESI $\dagger$ ). Thus, TW80 was selected as stabilizing agent. The best carbon dispersing performance was observed for a suspension containing $1 \mathrm{wt} \%$ of graphite and $0.5 \mathrm{wt} \%$ of surfactant in water (total mass $5 \mathrm{~kg}$ ). These standard suspensions were processed at different total pressures in the range from 10-70 MPa for 6 runs

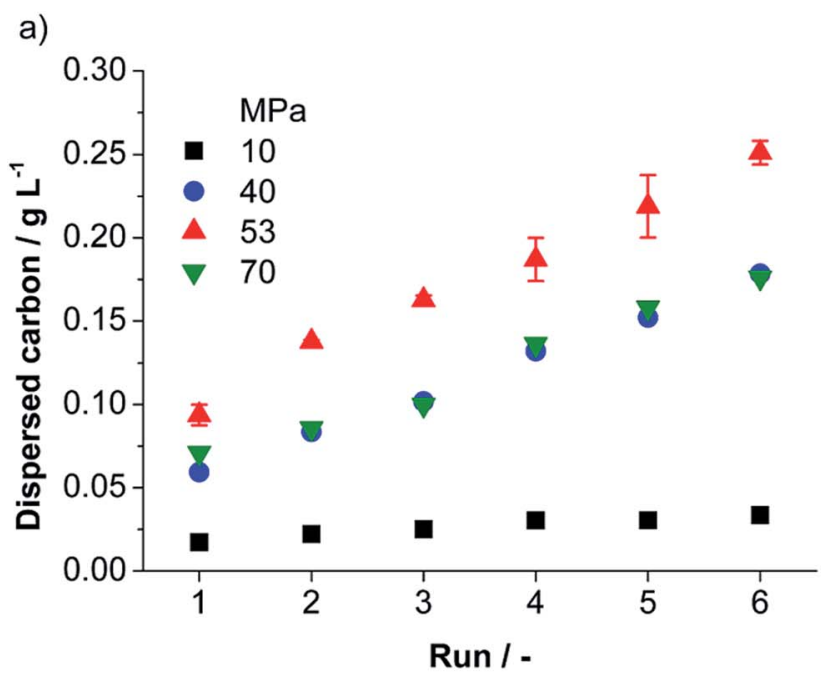

b)

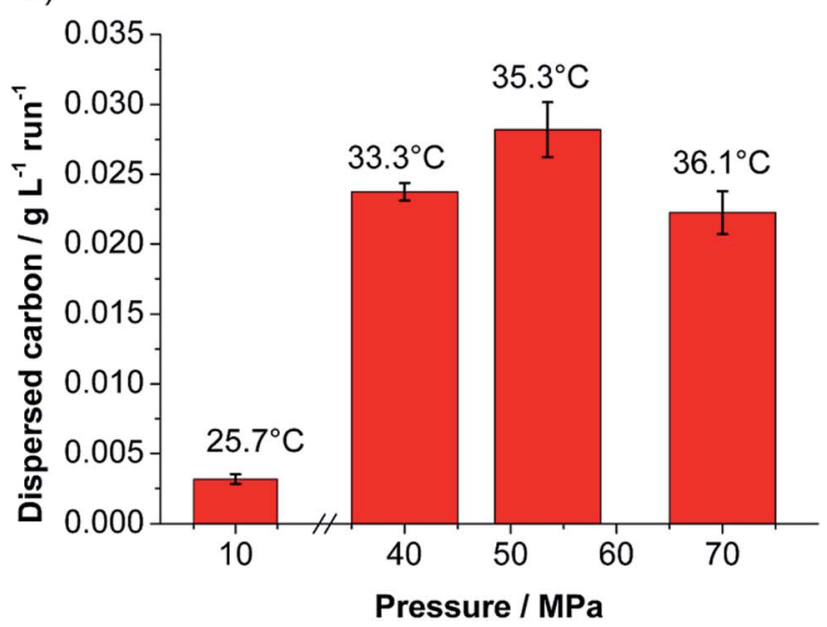

Fig. 2 (a) Dispersed carbon concentration obtained for standard suspension as a function of the number of batch runs for different applied pressure, (b) calculated concentration increase per run and average suspension temperature taken after each run. in batch mode. For all pressures investigated the dispersed carbon concentration increases linearly with the number of batch runs hinting for a continuous and homogenous delamination process (compare Fig. 2a).

The dispersed carbon concentration increases with higher nozzle pressure and reaches a maximum at $53 \mathrm{MPa}$ (see Fig. 2). The maximum dispersed carbon concentration obtained was $0.251 \mathrm{~g} \mathrm{~L}^{-1}$ after 6 runs and a related average concentration increase of $28.2 \mathrm{mg} \mathrm{L}^{-1}$ per run was achieved. For an increase of pressure from 53 to $70 \mathrm{MPa}$, however, a drop of concentration by roughly $20 \%$ is observed (see Fig. 2 ).

In the following we discuss possible delamination mechanisms to give an explanation for these results. Yi et al. investigated the delamination of hexagonal $\mathrm{BN}$, graphite and other layered materials in a high pressure nozzle experimentally and by Computational Fluid Dynamics (CFD) simulations and found that Reynolds shear stress, normal forces due to pressure release, particle-particle collisions and cavitation all contribute to the delamination. ${ }^{34,35}$ In our case the basic mechanisms should be similar although their relative importance might be shifted due to different nozzle geometries. A quantitative determination of the contributions of the different mechanisms to the delamination result would require an exact fluid mechanical analysis of the suspension flow in the HPH device. This is, however, beyond the frame of this paper, because here we focus on careful analysis of the obtained delaminated products. Thus, the discussion is limited to qualitative aspects of possible delamination mechanisms. We estimated the Reynolds number as function of flow rate for a nozzle diameter of $240 \mu \mathrm{m}$ and found that the Reynolds number is always $>10000$, i.e. a fully turbulent flow prevails. A higher nozzle pressure is directly coupled to higher flow rates and therefore to higher Reynolds numbers. The observed highly efficient delamination is in accordance with Liu et al.,${ }^{21}$ Paton et al. ${ }^{19}$ and Varrla et al. ${ }^{\mathbf{2 0}}$ who found for rotor-stator devices efficient graphite delamination for shear rates $>10000 \mathrm{~s}^{-1}$.

Cavitation in a high pressure microchannel flow was investigated by micro Particle Image Velocimetry ( $\mu$-PIV) and by CFD simulations. ${ }^{45,46}$ Even for a pressure of only $5 \mathrm{MPa}$ cavitation was observed by $\mu$-PIV. ${ }^{44}$ Moreover, it was observed that cavitation increases with higher pressure. ${ }^{46}$ Thus, we expect that in the whole investigated nozzle pressure range cavitation contributes to graphite delamination, if no valve pressure is applied. In $\mathrm{HPH}$ cavitation can be suppressed by applying counter pressure. Gothsch et al. observed complete elimination of cavitation for a counter pressure of $16 \mathrm{MPa}$ and a total pressure (nozzle pressure + counter pressure) of $52 \mathrm{MPa}{ }^{46}$ The pressure ratio corresponds to a Thoma number of 0.31 . The Thoma number is the ratio of the counter pressure to the total pressure. We performed graphite delamination experiments with applied counter pressure by closing the valve at the end of the expansion chamber and realized Thoma numbers up to 0.67 . The probability of cavitation decreases with increasing Thoma number. If the Thoma number is varied and the total pressure is kept constant, almost the same product concentration was achieved. Thus, besides cavitation also other mechanisms must contribute remarkably to graphite delamination in the used HPH device. 
The probability of graphite delamination due to selflubricating by collisions between feed particles increases with the collision kernel and with the square of the number concentration of feed particles in the suspension. In all experiments the feed concentration was the same. Thus, the collision rate increases directly proportional to the collision kernel. In the ESI $\dagger$ we calculated the collision kernel for particles $20 \mu \mathrm{m}$ in diameter (mean feed particle diameter) in turbulent flow. We found that the collision kernel and therefore the contribution of graphite delamination by self-lubrication increases by the factor of 3 if the nozzle pressure increases from 10 to $70 \mathrm{MPa}$, but the obtained product concentration increases by a factor of $\sim 10$ (Fig. 2b).

This qualitative discussion of the different mechanisms shows that the efficiency of graphite delamination should increase continuously with growing nozzle pressure. Thus, for the highest nozzle pressure $(70 \mathrm{MPa})$ the highest product concentration is expected. In contrast to our expectations the maximum of the dispersed carbon concentration was not observed at the highest pressure (70 $\mathrm{MPa})$, but rather at an intermediate pressure of $53 \mathrm{MPa}$ (see Fig. 2). A likely explanation can be given by taking agglomeration into account. Collisions between product particles or between a product and a feed particle can lead to agglomeration. As shown in the ESI $\uparrow$ also for that case the collision kernel and therefore the probability of collisions increases with growing nozzle pressure. Moreover, an increase in nozzle pressure is related to a rise of temperature (see Fig. 2b). Elevated temperatures may lead to destabilization of the suspension against agglomeration and re-stacking by desorption of the physical adsorbed surfactant on the graphene surface, ${ }^{47}$ or even preventing the surfactant to adsorb on the graphene surface. The argument of enhanced agglomeration for processing with a nozzle pressure of $70 \mathrm{MPa}$ is supported by results obtained for a cut size of $200 \mathrm{~nm}$, see ESI. $\dagger$ For a cut size of $200 \mathrm{~nm}$ the dispersed carbon concentration increases with growing nozzle pressure and the highest concentration is obtained as expected for $70 \mathrm{MPa}$. A comparison of the results in Fig. 2 and S3 (ESI $\dagger$ ) shows that at $70 \mathrm{MPa}$ indeed a higher amount of fine product particles is formed, but there is a depletion of particle sizes between 200 and $400 \mathrm{~nm}$. This can be explained by agglomeration of this size fraction and formation of agglomerate sizes $>400 \mathrm{~nm}$. Thus, these large particles are removed from the suspension during the centrifugation step (cut size $=400 \mathrm{~nm}$ ), finally resulting in a lower found carbon concentration in suspension.

To investigate transferability of the results to different feed materials also unmodified natural graphite was processed instead of the unmodified isostatic synthetic graphite under optimized conditions (nozzle pressure $53 \mathrm{MPa}$, no valve pressure). We found for the natural graphite feed material slightly higher production rates compared to the synthetic isostatic graphite (see $\mathrm{ESI}_{\dagger}^{\dagger}$ ). We account this to a smaller mean feed grain size of $3.5 \mu \mathrm{m}$ of the natural graphite compared to 15-20 $\mu \mathrm{m}$ mean feed size for the synthetic graphite. The total energy required for overcoming the van-der-Waals interaction and therefore for delamination increases with the surface area and therefore with the lateral dimension of the delaminated sheet. Thus, smaller flakes are easier to delaminate than larger ones.

\section{Evaluation of product quality by statistical Raman spectroscopy}

In previous studies we demonstrated for graphene production by stirred media delamination that processing parameters govern the obtained product quality and delamination behaviour. In particular, we found that the energy input influences the composition of the dispersed carbon phase, i.e. the content, layer thickness and quality of the delaminated flakes depend on the processing parameters. ${ }^{17,18}$ Therefore, FLG/SLG-yield and related defect characteristics of the samples processed by $\mathrm{HPH}$ have been determined by statistical Raman spectroscopy and are discussed in this section in dependence of process parameters.

A typical Raman spectrum consists of three main peaks, i.e. a D-, G- and 2D-peak. For the evaluation, we focus on the intensity ratio of $\mathrm{D} / \mathrm{G}$ and to the full widths at half maximum (FWHM) of the G- and 2D-peaks (G-FWHM and 2D-FWHM). The 2D-FWHM reflects the degree of delamination. Thus it can be used to distinguish between graphite $\left(>66 \mathrm{~cm}^{-1}\right.$, more than five layers of graphene), FLG (65-40 $\mathrm{cm}^{-1}, 2-5$ layers) and SLG $\left(<40 \mathrm{~cm}^{-1}\right.$, one layer of graphene)..$^{3,48-51}$ Note that the 2D-FWHM is enlarged by defects, edges and dopants so that the FLG and SLG contents derived from the 2D-FWHM distribution can be underestimated. However, as for graphene production large lateral dimensions and defect free product particles are of major importance, evaluation of the 2D-FWHM matches the purpose of determination of quality/degree of delamination.

The intensity ratio of the D- and G-peak (D/G ratio) increases inversely proportional to the measured graphite/graphene crystallite size $L_{\mathrm{a}}$ and is expressed by eqn (1) (Tuinstra-Koenig relation, $C_{\lambda}$ is a wavelength dependent constant). ${ }^{52-54}$

$$
\frac{I_{\mathrm{D}}}{I_{\mathrm{G}}} \propto \frac{C_{\lambda}}{L_{\mathrm{a}}}
$$

Cançado et $a l .{ }^{55}$ have demonstrated how this ratio is also influenced by the defect density, i.e. the in-plane damage of a graphene layer, which generally leads to larger values for $D / G$ with increasing number of defects per unit area (stage 1). For high defect densities the $\mathrm{D} / \mathrm{G}$ ratio decreases, if the defect concentration is further increased (stage 2). To distinguish between the low defect region (stage 1) and the high defect region (stage 2) the G-FWHM has to be evaluated. Stage 1 is attributed to G-FWHM values of $<30 \mathrm{~cm}^{-1}$ and stage 2 is attributed to G-FWHM $>30 \mathrm{~cm}^{-1}$. A comprehensive picture of sample quality regarding delamination and defects is thereby based on a combined discussion of 2D-FWHM, G-FWHM and $\mathrm{D} / \mathrm{G}$ ratio.

In the first step the influence of nozzle pressure (10-70 MPa) is evaluated for the final run (6) with respect to the 2D-FWHM cumulative distribution and yielded FLG-content (see Fig. 3a). Already for a nozzle pressure of $10 \mathrm{MPa}$ a suspension of well delaminated particles with a FLG content of $85 \%$ was obtained. 
The maximum FLG content is found for $40 \mathrm{MPa}$ with $94 \%$. For 53 and $70 \mathrm{MPa}$, respectively, FLG contents of $89 \%$ and $88 \%$ are found. The 2D-FWHM distribution in Fig. 3a also demonstrates a higher content of small values of 2D-FWHM for increasing pressure indicating a higher degree of delamination up to single layer graphene. For $53 \mathrm{MPa} \sim 0.5 \%$ of the evaluated Raman spectra indicated single layer graphene (for typically graphene spectra see ESI $\dagger$ ). Combining the FLG contents derived from 2D-FWHM distribution with the dispersed carbon concentration (see previous section Fig. 2) we obtain the FLG concentration (Fig. 3b). The highest FLG output after 6 runs was found for $53 \mathrm{MPa}$ followed by $40 \mathrm{MPa}$ with $0.223 \mathrm{~g} \mathrm{~L}^{-1}$ and 0.175 $\mathrm{g} \mathrm{L}^{-1}$, respectively.

The FLG production rate is calculated from the FLG concentration achieved after 6 runs: the flow rate to generate a pressure of 53 and $40 \mathrm{MPa}$, respectively, was 48 and $40 \mathrm{~L} \mathrm{~h}^{-1}$. Thus, it requires 6.2 and $7.5 \mathrm{~min}$ per run, respectively, to process a batch volume of $5 \mathrm{~L}$. Before each batch run the suspension was stirred in the holding tank for $15 \mathrm{~min}$ to cool it down to $18{ }^{\circ} \mathrm{C}$. Therefore, one batch run at 53 and $40 \mathrm{MPa}$,

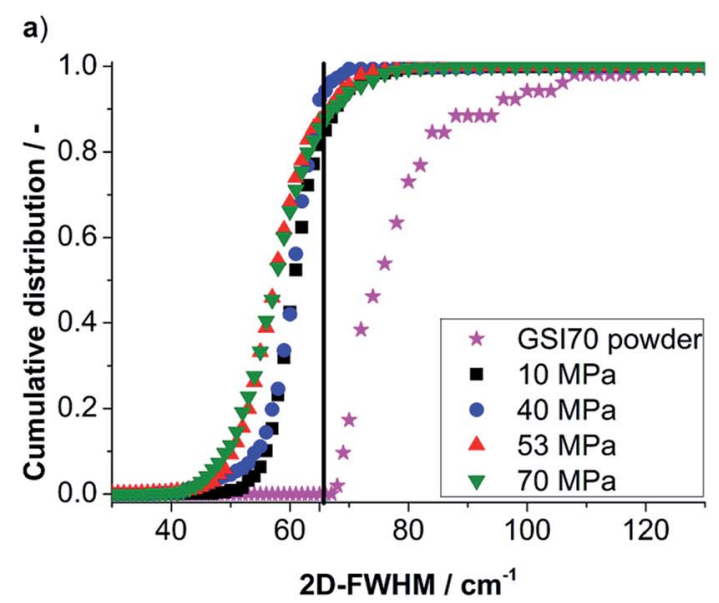

b)

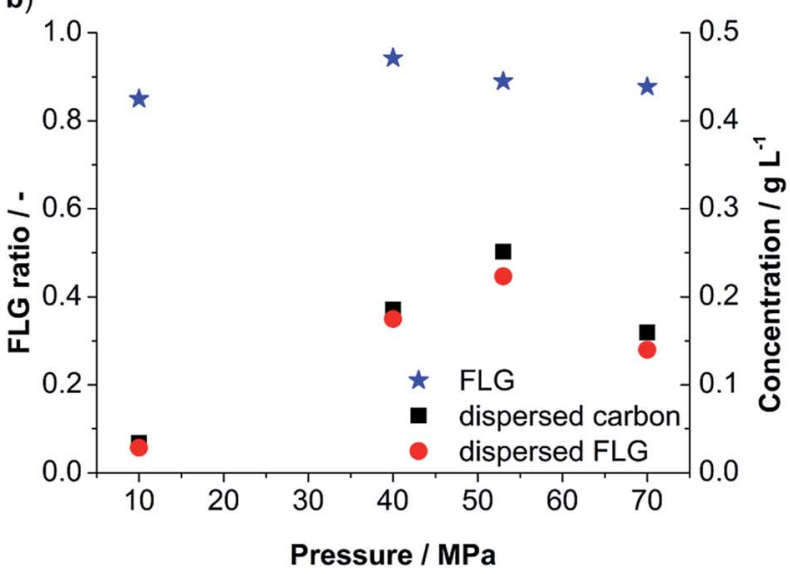

Fig. 3 (a) Cumulative distribution of $2 \mathrm{D}-\mathrm{FWHM}$ as a function of applied pressure for run 6 of standard suspensions. (b) Obtained FLG content (blue stars, left axis) and FLG-concentration calculated by combining the found FLG content with dispersed carbon concentration found by UV/Vis (red dots, right axis). respectively, takes 21.2 and 22.5 min and 6 runs require 130 and $135 \mathrm{~min}$ in total, respectively. Thus, the achieved FLG concentrations of $0.223 \mathrm{~g} \mathrm{~L}^{-1}(53 \mathrm{MPa})$ and $0.175 \mathrm{~g} \mathrm{~L}^{-1}(40 \mathrm{MPa})$, correspond to FLG production rates of $0.103 \mathrm{~g} \mathrm{~L}^{-1} \mathrm{~h}^{-1}(0.515 \mathrm{~g}$ $\left.\mathrm{h}^{-1}\right)$ and $0.078 \mathrm{~g} \mathrm{~L}^{-1} \mathrm{~h}^{-1}\left(0.390 \mathrm{~g} \mathrm{~h}^{-1}\right)$. It is expected that the FLG production rate can be increased considerably by numbering-up. The supplier of the used $\mathrm{HPH}$ device also provides a machine with 4 parallel nozzles operating at the same pressures as the used device.

For ultrasound-assisted graphite exfoliation FLG concentrations of about $2 \mathrm{~g} \mathrm{~L}^{-1}$ were achieved which is about one order of magnitude higher in comparison with our method. ${ }^{56}$ However, a direct comparison is not possible as the post-processing by centrifugation has a remarkable influence on the yielded concentration for top down processing. ${ }^{17,18}$ Khan et al. centrifuged the suspension with lower relative centrifugal force than in this work and therefore more product remained dispersed.

Furthermore, because of small batch volumes and long process times for ultrasound processing, the FLG production rate of our method is at least one order of magnitude higher in comparison with ultrasound processing. The results from sonication-assisted graphite exfoliation show that the "solubilitylimit" for FLG must be, however, much higher than the FLG concentration achieved in our work. Thus, we expect that we can increase the FLG concentration by either performing more batch runs or by increasing the graphite feed concentration.

The distribution of the number of graphene layers per particle can be determined from the 2D-FWHM distribution (compare Fig. 4a). According to (ref. 57-59) 2D-FWHM is inversely proportional to the number of graphene layers per particles. Thus, the number of layers per particle $N$ can be calculated by eqn (2) from the data shown in Fig. $3 a$.

$$
2 \mathrm{D}-\mathrm{FWHM}=71.5 \mathrm{~cm}^{-1}-\left(\frac{32.5 \mathrm{~cm}^{-1}}{N}\right)
$$

Evaluation of the 2D-FWHM distributions shown in Fig. 3a by eqn (2) reveals that three-layer graphene is the main

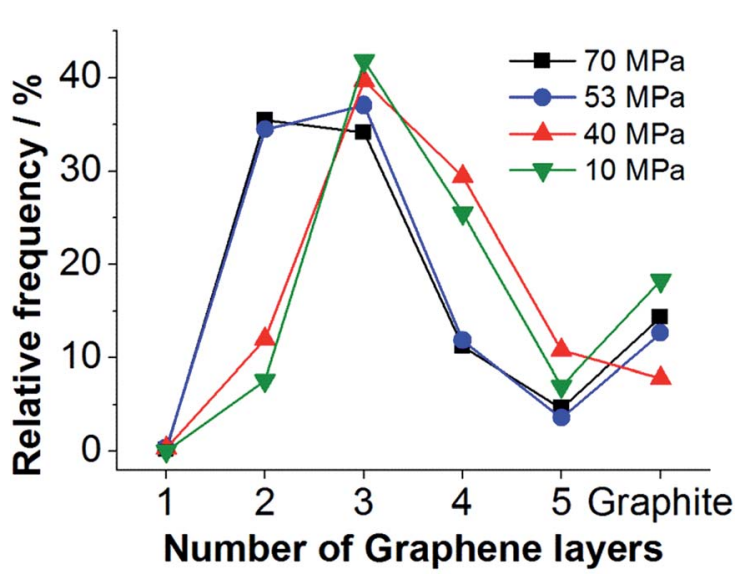

Fig. 4 Distribution of the number of graphene layers per particle calculated from the obtained 2D-FWHM distribution for graphene suspensions processed by HPH at different nozzle pressures. 
component of the FLG suspensions processed at lower pressures, see Fig. 4. In the samples processed with a nozzle pressure of 53 and $70 \mathrm{MPa}$, respectively, bilayers appear with a similar frequency as three-layers. Thus, for higher nozzle pressure a better degree of delamination is achieved.

A detailed view on the influence of processing parameters on product yield and quality is provided by plotting the mean values of 2D-, G-FWHM and D/G against the pressure. For the processing conditions that lead to the highest FLG-yield (53 MPa) 2D-, G-FWHM and D/G are also plotted against the number of runs (compare Fig. 5a and c). 2D-FWHM decreases slightly for higher pressures indicating a better delamination. The mean G-FWHM is below $30 \mathrm{~cm}^{-1}$ for all applied pressures showing that the products are in the low defective region. The $\mathrm{D} / \mathrm{G}$ ratio is always below 1 and reaches a maximum for $53 \mathrm{MPa}$ emphasizing the lowest mean average lateral dimension, even lower than for $70 \mathrm{MPa}$ (compare Fig. 5a). Note: values for $\mathrm{D} / \mathrm{G}<1$ can be predominantly related to edge effects for G-FWHM values $<30 \mathrm{~cm}^{-1} \cdot{ }^{60,61}$ In Fig. $5 \mathrm{c}$ the development of 2D-FWHM, G-FWHM and D/G ratio over 6 runs is shown for a sample processed at $53 \mathrm{MPa}$. Fig. 5c illustrates that the values for
G-FWHM and D/G ratio change only within the range of the obtained standard deviations. From the values for 2D-FWHM for higher number of runs a slight tendency for a better delamination can be seen. The process yields particles almost exclusively in the low defective regime.

For both, applied pressure and number of performed runs a property map, i.e. a plot of $2 \mathrm{D}-\mathrm{FWHM}$ vs. D/G, was created to show clearly the influence of processing parameters on the yielded quality (compare Fig. 5b and d). Fluctuations of the values obtained for the six runs at $53 \mathrm{MPa}$ pressure are within the standard deviations. Only a slide tendency of better delamination for a growing number of runs is observed, expressed by lower 2D-FWHM and lower D/G ratios (compare Fig. 5d). A comparison of Fig. $5 \mathrm{~b}$ and d shows that the pressure has a larger influence on the product quality than the number of performed batch runs. Better delaminated, smaller particles are obtained for higher applied pressure as found by statistical Raman spectroscopy (also compare Fig. 3a and 4) showing a higher content of low 2D-FWHM values. Interestingly, the experiment performed with $70 \mathrm{MPa}$ yielded larger particles compared to $53 \mathrm{MPa}$ since Raman mapping showed a smaller
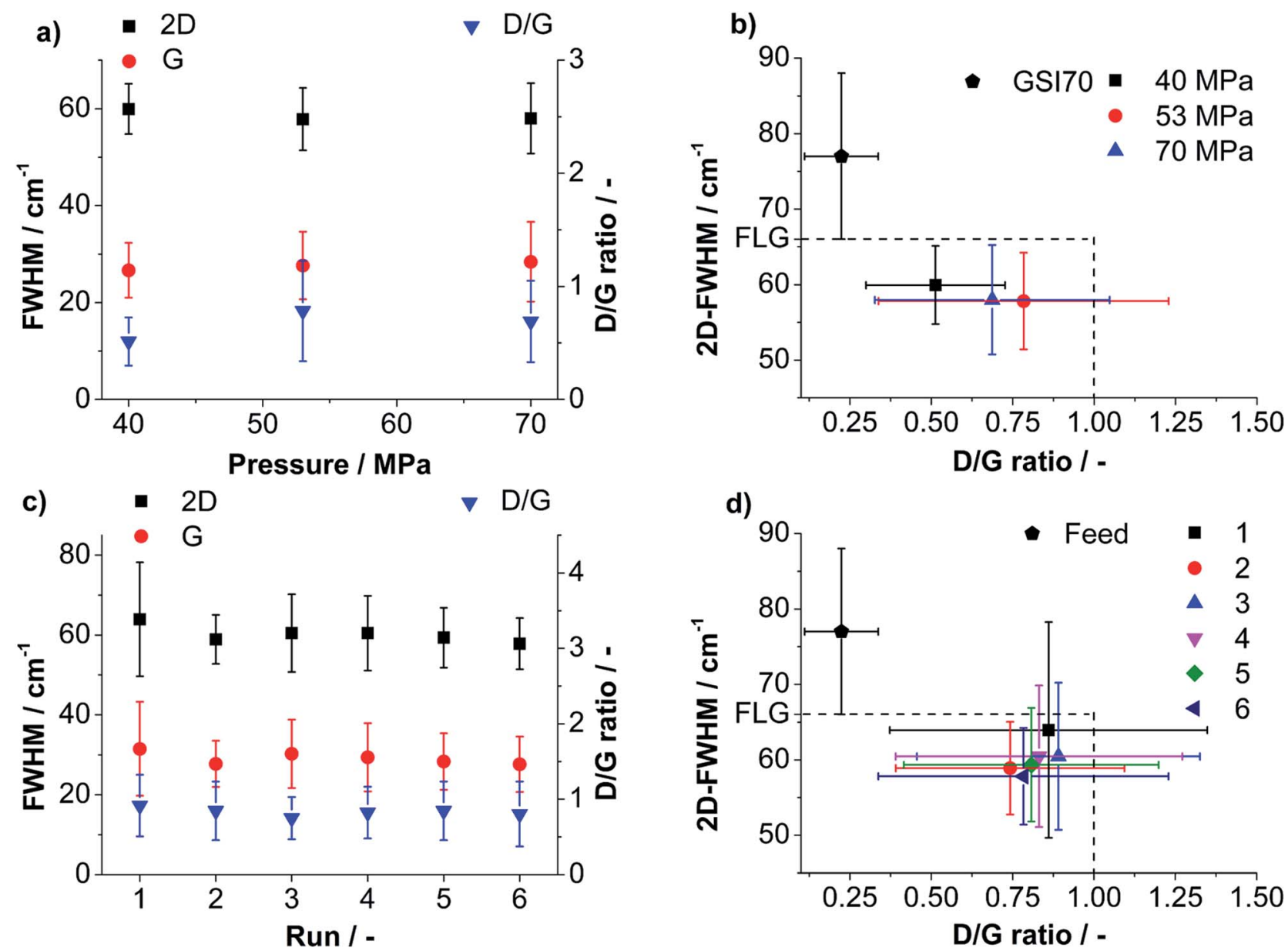

Fig. 5 (a) Mean values for evaluated Raman statistics 2D-, G-FWHM (left axes) and D/G ratio (right axes) as a function of applied pressure for run 6 of standard suspensions, (b) 2D-FWHM vs. D/G ratio for run 6 of standard suspensions processed at different pressures, (c) mean values for evaluated Raman statistics 2D-, G-FWHM (left axes) and D/G ratio (right axes) as a function of the number of batch-runs for a standard suspension processed at $53 \mathrm{MPa}$, (d) 2D-FWHM vs. D/G ratio for standard suspensions processed over 6 batch runs with $53 \mathrm{MPa}$. 
D/G ratio accompanied by a similar G-FWHM. This finding corresponds well to the UV/Vis measurements. In agreement with UV/Vis measurements the process produces a high content of FLG particles whereby a slight improvement of the overall delamination with growing applied pressure is observed.

\section{Morphology of processed particles}

The degree of delamination derived from statistical Raman spectroscopy was validated by AFM, because it measures the flake thickness and lateral dimensions directly. As discussed in literature any residual surfactants on a graphene surface disturb AFM imaging., ${ }^{9,19}$ Only few molecules on the graphene particles can create considerable fluctuations of measured heights obtained in AFM. For this reason AFM investigation was performed on a sample processed in NMP, as good results in removal of NMP could be achieved. Processing was performed with a nozzle pressure of $53 \mathrm{MPa}$ and without valve pressure at the standard concentration of $1 \mathrm{wt} \%$ GSI70. The obtained suspension (centrifuged to cut size $=400 \mathrm{~nm}$ ) was analyzed by statistical Raman spectroscopy and AFM.

A total of 7 AFM images, each spanning $5 \times 5 \mu \mathrm{m}^{2}$ were evaluated. 685 flakes were detected and a mean height of $2.4 \mathrm{~nm}$ was obtained over all flakes. Diameters obtained range from $20-$ $580 \mathrm{~nm}$ with a mean equivalent disc diameter of $34.9 \mathrm{~nm}$ (see Fig. 6). The typical flake thickness for single layer graphene on an oxidized silicon wafer ( $300 \mathrm{~nm} \mathrm{SiO}_{2}$-layer) is about $1 \mathrm{~nm}$, the interlayer distance for 2 graphene layers is $\sim 0.35 \mathrm{~nm}$ and the intrinsic layer thickness is $\sim 0.34 \mathrm{~nm}$ yielding a step height of $\sim 0.69 \mathrm{~nm}$ per layer after the first layer. ${ }^{1,2}$ A measured height $<3.8 \mathrm{~nm}$ thereby corresponds to FLG ( $\leq 5$ layers). The FLG content was determined to be $74.4 \%$ as obtained by AFM imaging (see Fig. 6) with a mean number of three layers.

In excellent agreement to AFM, statistical Raman spectroscopy yields a FLG content of $75.9 \%$ proving that the FLG contents derived from statistical Raman spectroscopy are highly reliable. Moreover, for the sample processed in NMP statistical Raman spectroscopy yields a mean D/G ratio of 0.64 and GFWHM of $29.3 \mathrm{~cm}^{-1}$ indicating that the flakes contain only few defects.

The obtained mean $\mathrm{D} / \mathrm{G}$ ratio for the aqueous surfactantbased suspensions processed at 40-70 MPa ranged from 0.520.78 . From the similar $\mathrm{D} / \mathrm{G}$ values it can be concluded that the lateral diameter distribution of the NMP based sample should be in the same range as for the aqueous samples.

The sample processed in NMP with a nozzle pressure of 53 MPa was also investigated by AFM Raman spectroscopy colocalization, see ESI. $\dagger$ An advantage of this method is that height profile and Raman spectrum are measured exactly at the same position on the wafer. In Fig. 7 a survey AFM image, an enlarged section of this image and extracted height profiles and Raman spectra of selected flakes are shown.

The co-localized experiments yield an even higher FLG content (86.3\%) in comparison to the independent AFM and Raman spectroscopic investigations (about 75\% FLG), see ESI. $\dagger$ This result shows that Raman spectroscopy indeed gives a worst-case scenario regarding the FLG-content, e.g. the real
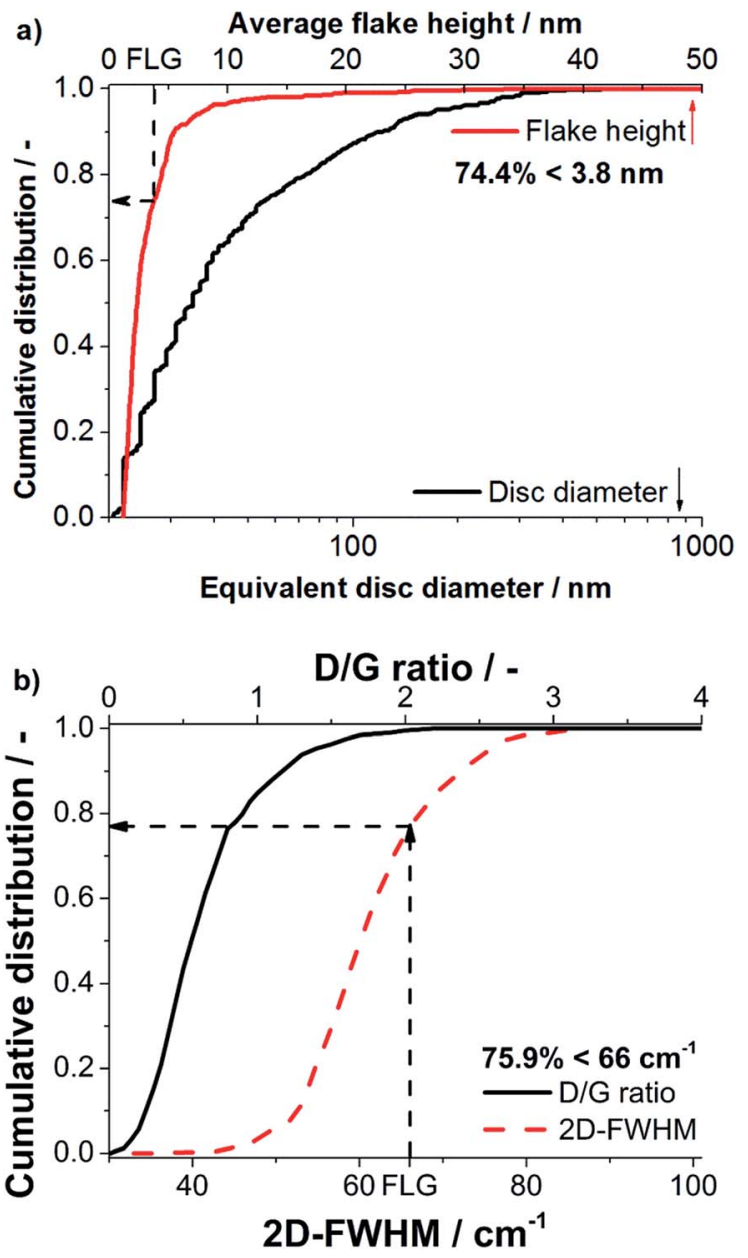

Fig. 6 (a) Cumulative distributions of the flake height and equivalent disc diameter obtained by AFM for a NMP based suspension processed at $53 \mathrm{MPa}$, (b) cumulative distribution of the D/G ratio and 2D-FWHM determined by a statistical Raman spectroscopic analysis.

product quality regarding delamination degree is even better than indicated by Raman spectroscopy alone.

\section{Analytical ultracentrifugation}

AUC-SV experiments were performed with supernatants of samples centrifuged to the standard cut size value of $400 \mathrm{~nm}$ to study their hydrodynamic properties. Due to its absolute measurement and fractioning principle, AUC provides highly accurate and quantitative information on the size of particles and is therefore perfectly suited to study complex and polydisperse samples in liquid media. As shown previously in a work on graphene oxide monolayers, the sedimentation coefficient distribution can be converted to a lateral size distribution in case of a constant sheet thickness. ${ }^{39}$ The major advantage of AUC is that the particle size is analyzed directly in solution. Therefore, no wafer preparation and potential related problems as drying/size exclusion effects are witnessed. For a first comparison of the different pressures and batch runs, we did not pursue such a conversion because a distribution of the number of graphene layers per particle is present in each 
a)

b)

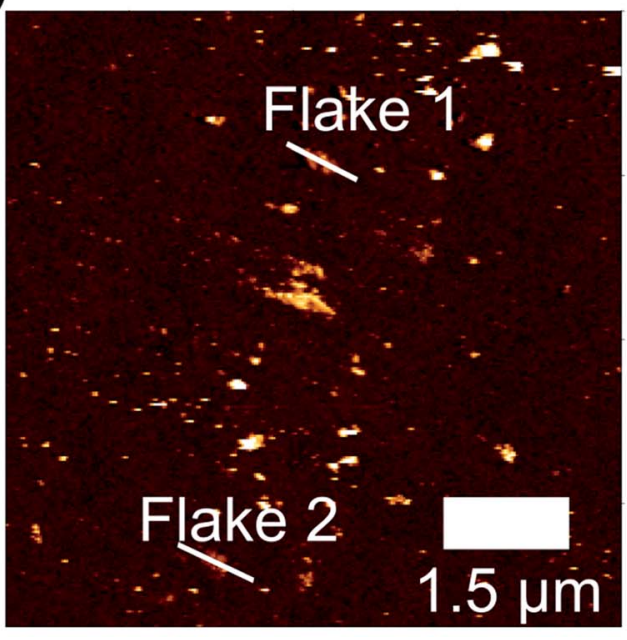
spectra and (d) height profiles of the marked flakes in (b). c)

\section{$10 \mathrm{~nm}$}
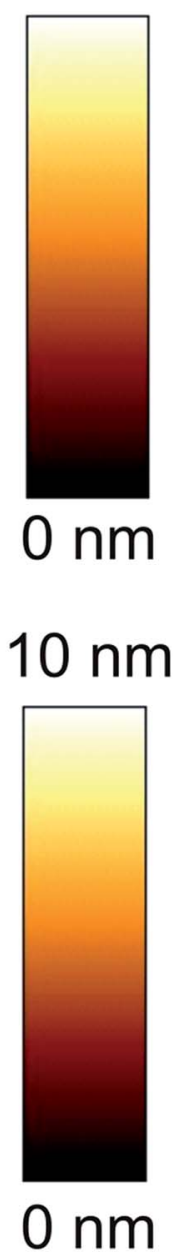

d)
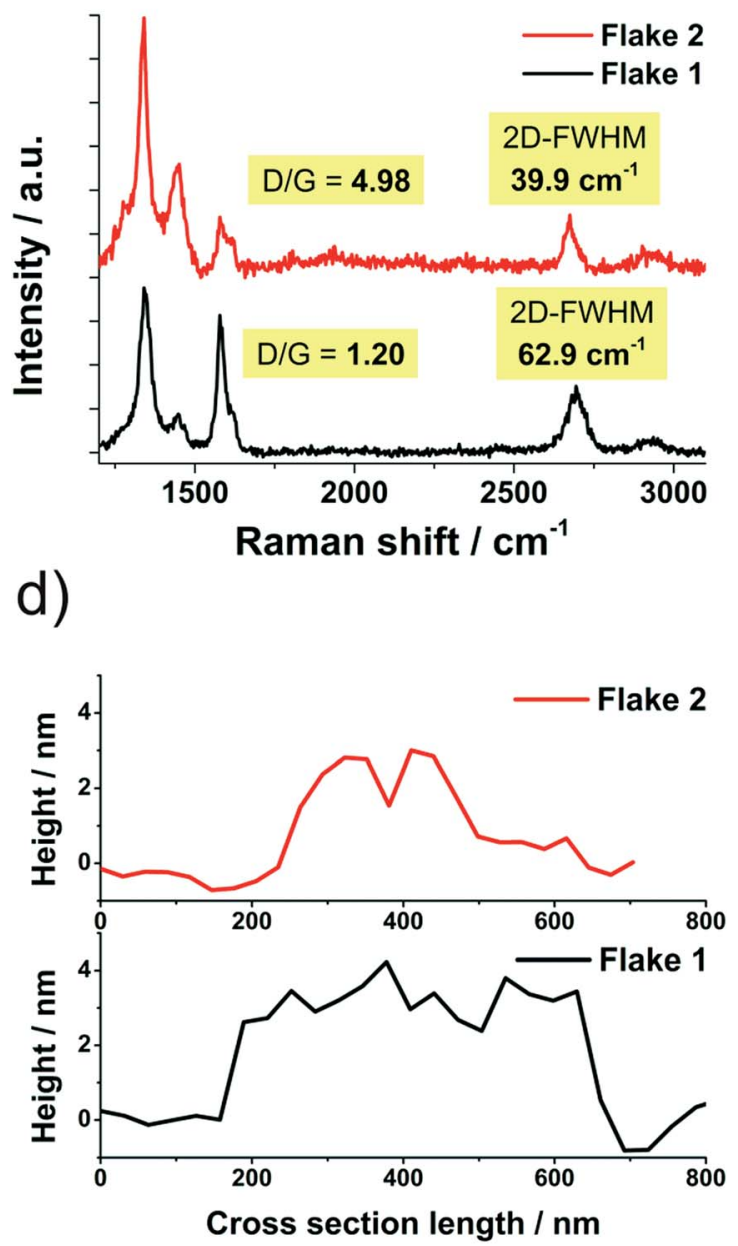

Fig. 7 (a) Survey AFM image of a sample processed in NMP with 53 MPa nozzle pressure, (b) zoom AFM image of the marked area in (a), (c) Raman

sample. Instead we use the sedimentation coefficient distribution, which is the primary quantity of an AUC experiment to assess the morphology of the graphite structures. The sedimentation coefficient scales linearly with the lateral diameter and sheet thickness.

We investigated samples processed at three different nozzle pressures (40, 50 and $70 \mathrm{MPa}$ ) and different run counts. The results of the AUC analysis are presented in Fig. 8.

As it can be seen in Fig. 8a, structures with sedimentation coefficients between 100 and 90000 sved were found by AUC. The upper boundary is equivalent to a sedimentation equivalent spherical particle size of $399 \mathrm{~nm}$, which agrees perfectly to the adjusted cut size of $400 \mathrm{~nm}$. For all pressures the sedimentation coefficient and thus the particle size decreases with the number of runs. However, for run six at $40 \mathrm{MPa}$ larger structures are again detected, an effect which may be attributed to minor fluctuations during processing (compare Fig. 5c and d). We further compared the final batch run six at three pressures, see Fig. 8 b. For $40 \mathrm{MPa}$ by far the largest structures were found. The samples produced at 53 and $70 \mathrm{MPa}$ have a similar distribution but the sample processed at $70 \mathrm{MPa}$ contains some more fines and also more course particles compared to the sample processed at $53 \mathrm{MPa}$. This result is again in good agreement to the data obtained by the statistical Raman spectroscopy and UV-Vis measurements.

Since a near quantitative yield of FLG (>94\%) was found for the $40 \mathrm{MPa}$ sample, we converted the sedimentation coefficient distribution of run five to a lateral diameter distribution according to the methodology described previously in detail by Walter and Nacken et al. ${ }^{39}$ The results of the diameter analysis are shown in Fig. 9. The maximum and minimum sheet diameters depend on the number of layers used for the calculation. We used sheet thicknesses of one, three and five layers to estimate the boundary values for the lateral sizes. The true lateral diameter distribution of the sample processed with 40 $\mathrm{MPa}$ is expected to range from $60-4000 \mathrm{~nm}$ as the main fraction of product particles consists of three layers $(n=3)$ as found by statistical Raman spectroscopy. 

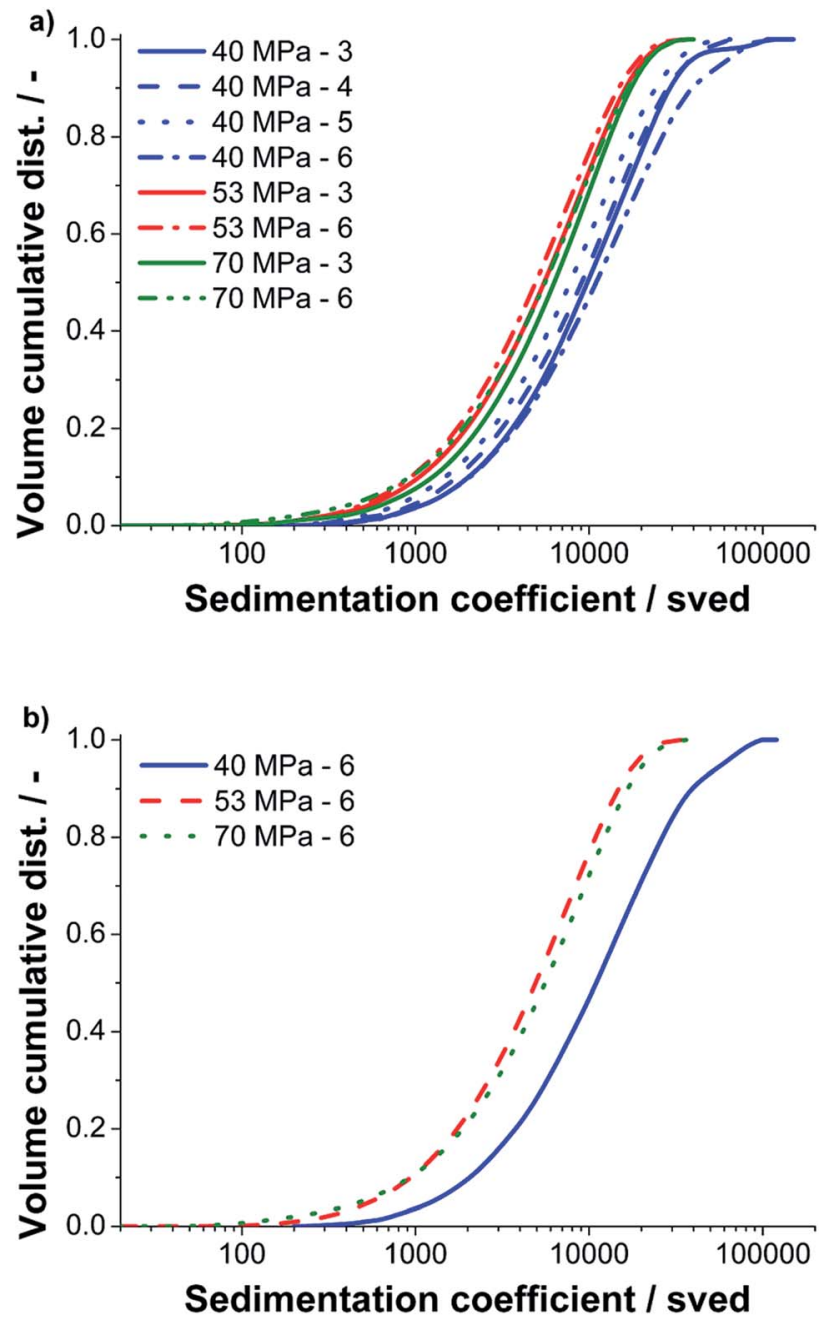

Fig. 8 (a) Volume cumulative distributions of the sedimentation coefficient for samples produced at different pressures and with different run counts, (b) selected sedimentation coefficient distributions for the sixth run. For sheets of a constant thickness, the lateral diameter increases linearly with the sedimentation coefficient.

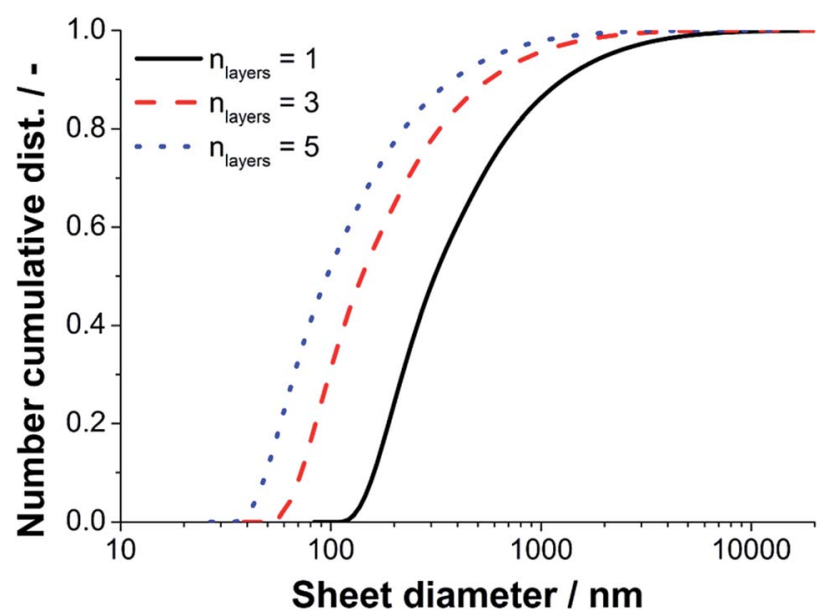

Fig. 9 Cumulative number weighted distribution of the lateral sheet diameter for run five at $40 \mathrm{MPa}$. The minimum diameter is given by sheets with a thickness of five graphene layers and the maximum diameter is given assuming single layer graphene.

\section{Conclusions}

FLG suspensions of good quality with respect to degree of delamination and few in-plane defects can be prepared on large scale by graphite delamination in an industrial high pressure homogenizer. The overall adjusted pressure was found as the dominant processing parameter. Turbulent shear stress, cavitation and collisions between the feed particles contribute to delamination and all contributions are expected to increase with growing nozzle pressure.

The maximum achieved FLG concentration is $0.223 \mathrm{~g} \mathrm{~L}^{-1}$ corresponding to a production rate of $0.106 \mathrm{~g} \mathrm{~L}^{-1} \mathrm{~h}^{-1}$ (about 0.5 $\mathrm{g} \mathrm{h}^{-1}$ ) and was obtained for $53 \mathrm{MPa}$. Moreover, for $53 \mathrm{MPa}$ up to $\sim 0.5 \%$ single layer graphene was found. For all investigated pressures the FLG content in the centrifuged samples is at least $76 \%$. Up to $94 \%$ of FLG were detected for 40 MPa and 6 batch runs. Two- and three-layer graphene were found as the main components of the FLG suspensions processed at pressures of 53 and $70 \mathrm{MPa}$, whereas for lower pressure three-layer graphene is the dominant species. This result clearly demonstrates that a better degree of delamination is achieved with higher nozzle pressure. A homogenous delamination process was demonstrated by the intensive analytical study of six batch runs processed in sequence with a nozzle pressure of $53 \mathrm{MPa}$. Particles with lateral dimensions ranging from 40-1000 nm (disc equivalent diameter) were detected by AFM analysis of an NMP processed sample. The obtained FLG-content from statistical Raman spectroscopy showed high reliability and matched the content determined by AFM. Co-localized AFM and Raman spectroscopic investigations reveal that the real FLG content can even be higher than indicated by Raman spectroscopy alone. Thus, the FLG contents derived from statistical Raman spectroscopy have to be regarded as lower limit values. We further demonstrated the potential of analytical ultracentrifugation for the evaluation of lateral particle size directly in solution.

\section{Acknowledgements}

This work was supported financially by the German Science Foundation (DFG), Collaborative Research Centre SFB953: "Synthetic Carbon Allotropes". The authors would like to thank Netzsch Feinmahltechnik GmbH, Germany for offering the possibility on conducting this research on a high pressure homogenizer Omega ${ }^{860}$. Many thanks go to the student helpers Yusra Hambal and Maximilian Uttinger for support in AFM and experimental work and our colleagues C. Konnerth and S. Romeis for scientific discussions.

\section{References}

1 K. S. Novoselov, A. K. Geim, S. V. Morozov, D. Jiang, Y. Zhang and S. V. Dubonos, et al., Science, 2004, 5696, 666.

2 K. S. Novoselov, D. Jiang, F. Schedin, T. J. Booth, V. V. Khotkevich and S. V. Morozov, et al., Proc. Natl. Acad. Sci. U. S. A., 2005, 30, 10451.

3 A. K. Geim and K. S. Novoselov, Nat. Mater., 2007, 3, 183. 
4 S. V. Morozov, K. S. Novoselov, M. I. Katsnelson, F. Schedin, D. C. Elias and J. A. Jaszczak, et al., Phys. Rev. Lett., 2008, 1, 16602.

5 K. I. Bolotin, K. J. Sikes, Z. Jiang, M. Klima, G. Fudenberg and J. Hone, et al., Solid State Commun., 2008, 9-10, 351.

6 R. R. Nair, P. Blake, A. N. Grigorenko, K. S. Novoselov, T. J. Booth and T. Stauber, et al., Science, 2008, 5881, 1308.

7 M. J. Allen, V. C. Tung and R. B. Kaner, Chem. Rev., 2009, 1, 132.

8 Y. Zhu, S. Murali, W. Cai, X. Li, J. W. Suk and J. R. Potts, et al., Adv. Mater., 2010, 35, 3906.

9 Y. Hernandez, V. Nicolosi, M. Lotya, F. M. Blighe, Z. Sun and S. De, et al., Nat. Nanotechnol., 2008, 9, 563.

10 M. Lotya, P. J. King, U. Khan, S. De and J. N. Coleman, ACS Nano, 2010, 6, 3155.

11 L. Guardia, M. J. Fernández-Merino, J. I. Paredes, P. SolísFernández, S. Villar-Rodil and A. Martínez-Alonso, et al., Carbon, 2011, 5, 1653.

12 I. Jeon, H. Choi, S. Jung, J. Seo, M. Kim and L. Dai, et al., J. Am. Chem. Soc., 2013, 135, 1386.

13 Y. Lv, L. Yu, C. Jiang, S. Chen and Z. Nie, RSC Adv., 2014, 26, 13350.

14 Y. Yao, Z. Lin, Z. Li, X. Song, K. Moon and C. Wong, J. Mater. Chem., 2012, 27, 13494.

15 W. Zhao, M. Fang, F. Wu, H. Wu, L. Wang and G. Chen, J. Mater. Chem., 2010, 28, 5817.

16 C. Knieke, A. Berger, M. Voigt, R. N. K. Taylor, J. Röhrl and W. Peukert, Carbon, 2010, 11, 3196.

17 C. Damm, T. J. Nacken and W. Peukert, Carbon, 2015, 81, 284.

18 T. J. Nacken, C. Damm, H. Xing, A. Rüger and W. Peukert, Nano Res., 2015, DOI: 10.1007/s12274-014-0694-6.

19 K. R. Paton, E. Varrla, C. Backes, R. J. Smith, U. Khan and A. O'Neill, et al., Nat. Mater., 2014, 6, 624.

20 E. Varrla, K. R. Paton, C. Backes, A. Harvey, R. J. Smith and J. McCauley, et al., Nanoscale, 2014, 20, 11810.

21 L. Liu, Z. Shen, M. Yi, X. Zhang and S. Ma, $R S C A d v$., 2014, 69, 36464.

22 Z. Wang, J. Liu, W. Wang, H. Chen, Z. Liu, Q. Yu, H. Zeng and L. Sun, Chem. Commun., 2013, 49, 10835.

23 K. Parvez, Z.-S. Wu, R. Li, X. Liu, R. Graf, X. Feng and K. Müllen, J. Am. Chem. Soc., 2014, 136, 6083.

24 G. Brookman, Biotechnol. Bioeng., 1974, 3, 371.

25 G. Narsimhan and P. Goel, J. Colloid Interface Sci., 2001, 2, 420.

26 S. Mohan and G. Narsimhan, J. Colloid Interface Sci., 1997, 1, 1.

27 P. R. Mishra, L. A. Shaal, R. H. Müller and C. M. Keck, Int. J. Pharm., 2009, 1-2, 182.

28 J. Salazar, A. Ghanem, R. H. Müller and J. P. Möschwitzer, Eur. J. Pharm. Biopharm., 2012, 1, 82.

29 F. J. Tölle, M. Fabritius and R. Mülhaupt, Adv. Funct. Mater., 2012, 6, 1136.

30 A. Appel, R. Thomann and R. Mülhaupt, Polymer, 2012, 22, 4931.

31 B. Schlüter, R. Mülhaupt and A. Kailer, Tribol. Lett., 2014, 1, 353.

32 M. Yi, Z. Shen, X. Zhang and S. Ma, Appl. Phys. Lett., 2011, 99, 123112.
33 J. Li, Z. Shen, S. Ma, X. Zhang and Y. Xing, Sci. China: Technol. Sci., 2012, 55, 2815.

34 M. Yi, Z. Shen, W. Zhang, J. Zhu, L. Liu, S. Liang, X. Zhang and S. Ma, Nanoscale, 2013, 5, 10660.

35 M. Yi, Z. Shen and J. Zhu, Chin. Sci. Bull., 2014, 59, 1794.

36 S. Liang, Z. Shen, M. Yi, L. Liu, X. Zhang, C. Cai and S. Ma, J. Nanosci. Nanotechnol., 2015, 15, 2686.

37 S. Eigler, F. Hof, M. Enzelberger-Heim, S. Grimm, P. Müller and A. Hirsch, J. Phys. Chem. C, 2014, 14, 7698.

38 J. M. Englert, P. Vecera, K. C. Knirsch, R. A. Schäfer, F. Hauke and A. Hirsch, ACS Nano, 2013, 6, 5472.

39 J. Walter, T. J. Nacken, C. Damm, T. Thajudeen, S. Eigler and W. Peukert, Small, 2015, 11, 814.

40 S. Bhattacharyya, P. Maciejewska, L. Börger, M. Stadler, A. Gülsün and H. Cicek, et al., Prog. Colloid Polym. Sci., 2006, 131, 9.

41 J. Walter, K. Löhr, E. Karabudak, W. Reis, J. Mikhael and W. Peukert, et al., ACS Nano, 2014, 9, 8871.

42 P. Schuck and P. Rossmanith, Biopolymers, 2000, 5, 328.

43 M. Lotya, Y. Hernandez, P. J. King, R. J. Smith, V. Nicolosi and L. S. Karlsson, et al., J. Am. Chem. Soc., 2009, 10, 3611.

44 M. Yi, Z. Shen, X. Zhang and S. Ma, J. Phys. D: Appl. Phys., 2013, 2, 25301.

45 S. Beinert, T. Gothsch and A. Kwade, Chem. Eng. Sci., 2015, 123, 197.

46 T. Gothsch, C. Schilcher, C. Richter, S. Beinert, A. Dietzel, S. Büttgenbach and A. Kwade, Microfluid. Nanofluid., 2015, 18, 121.

47 Z. Sun, S. Pöller, X. Huang, D. Guschin, C. Taetz and P. Ebbinghaus, et al., Carbon, 2013, 64, 288.

48 J. M. Englert, J. Röhrl, C. D. Schmidt, R. Graupner, M. Hundhausen and F. Hauke, et al., Adv. Mater., 2009, 42, 4265.

49 S. Lee, K. Lee and Z. Zhong, Nano Lett., 2010, 11, 4702.

50 Y. Hao, Y. Wang, L. Wang, Z. Ni, Z. Wang and R. Wang, et al., Small, 2010, 2, 195.

51 S. Chen, W. Cai, R. D. Piner, J. W. Suk, Y. Wu and Y. Ren, et al., Nano Lett., 2011, 9, 3519.

52 F. Tuinstra and J. Koenig, J. Compos. Mater., 1970, 4, 494.

53 F. Tuinstra and L. Koenig, J. Chem. Phys., 1970, 3, 1126.

54 A. Jorio, E. H. Martins Ferreira, M. V. O. Moutinho, F. Stavale, C. A. Achete and R. B. Capaz, Phys. Status Solidi $B, 2010,247,2980$.

55 L. G. Cançado, A. Jorio, E. H. M. Ferreira, F. Stavale, C. A. Achete and R. B. Capaz, et al., Nano Lett., 2011, 8, 3190.

56 U. Khan, H. Porwal, A. O'Neill, K. Nawaz, P. May and J. N. Coleman, Langmuir, 2011, 27, 9077.

57 D. S. Lee, C. Riedl, B. Krauss, K. von Klitzing, U. Starke and H. J. Smet, Nano Lett., 2008, 8, 4320.

58 A. C. Ferrari, J. C. Meyer, V. Scardaci, C. Casiraghi, M. Lazzeri, F. Mauri, S. Piscanec, D. Jiang, K. S. Novoselov, S. Roth and A. K. Geim, Phys. Rev. Lett., 2006, 97, 187401.

59 D. Graf, F. Molitor, K. Ensslin, C. Stampfer, A. Jungen, C. Hierold and L. Wirtz, Nano Lett., 2007, 7, 238.

60 A. K. Gupta, T. J. Russin, H. R. Gutiérrez and P. C. Eklund, ACS Nano, 2008, 1, 45.

61 C. Casiraghi, A. Hartschuh, H. Qian, S. Piscanec, C. Georgi and A. Fasoli, et al., Nano Lett., 2009, 4, 1433. 